\title{
INSULIN RESISTANCE AND LIPID DISORDERS IN PRE-PUBERTAL CHILDREN BORN SMALL FOR GESTATIONAL AGE
}

\author{
A. Kapitsinou ${ }^{1}$, C. Tsendidis ${ }^{2}$, D. Kyriakou ${ }^{1}$, A. Foteinou ${ }^{3}$, A. Drakatos ${ }^{3}$, A. Garoufi $^{1}$ \\ ${ }^{1}$ 2nd Department of Pediatrics, P\&A Kyriakou Childrens Hospital, University of Athens, Medical School, \\ Athinai, ${ }^{2}$ Department of Endocrinology, General Hospital of Nicaea, Piraeus, ${ }^{3}$ Biochemistry Laboratory, \\ P\&A Kyriakou Childrens Hospital, Athínai, Greece
}

Background and aims: Epidemiological studies indicate that children with intrauterine growth retardation (IUGR) are at increased risk of developing metabolic syndrome and cardiovascular disease in adult life. The aim of this prospective study was to assess the prevalence of insulin resistance and lipid disorders in children with IUGR.

Methods: The study population consisted of 54 pre-pubertal healthy children (median age: 7years), 29 with IUGR and 25 born appropriate for gestational age (AGA). IUGR children had an uncomplicated neonatal period. A complete lipid profile, glucose and insulin were assessed in a fasting blood sample. Insulin resistance was evaluated using the fasted glucose to insulin ratio (Gl/Ins $\leq 6$ was considered abnormal), HOMA-IR and HOMA- $\beta$. Statistical analysis was performed using STATA 11.0. $\mathrm{P}<0.05$ was considered significant.

Results: Six out 29 IUGR (20.4\%) and none of AGA children had a ratio Gl/Ins $\leq 6$. HOMA-IR and HOMA$\beta$ were significantly higher in IUGR group $(1.26 \pm 0.86$ vs $0.92 \pm 0.51, p<0.05$ and $119.23 \pm 59.11$ vs 93.67 $34.09, \mathrm{p} 0.0312$, respectively). LDL-C concentrations were higher in IUGR $(\mathrm{p}=0.05)$ and triglycerides in AGA $(\mathrm{p}=0.047)$ children. HDL-Cholesterol, TSH, T4, the ratio waist/hip circumference and arterial pressure were similar in two groups. Classification of children regarding BMI, revealed no significant difference in all parameters apart HOMA- $\beta$ that was higher in the group of overweight and obese $(\mathrm{p}=0.004)$.

Conclusions: Children with IUGR are prone to develop insulin resistance thus diabetes type II; and lipid disorders comparing with those born AGA, independently of BMI. The ratio Gl/Ins, HOMA-IR and HOMA$\beta$ may be useful for detecting children at risk. 